\title{
INVESTIGACIONES
}

\section{La formación inicial docente en Chile: Tensiones entre políticas de apoyo y control}

\author{
Initial teachers' education in Chile: tensions between policies of support and control \\ Formação inicial de professores no Chile: Tensões entre política de apoio e controle
}

\author{
Beatrice Ávalos
}

Centro de Investigación Avanzada en Educación, Universidad de Chile

Telf.: (562)29782762. Correo electrónico: bavalos@ terra.cl

\begin{abstract}
RESUMEN
El artículo examina iniciativas de política nacional que han afectado a la formación inicial docente y su efectividad en los últimos quince años. Para ello se utilizan las categorías formuladas por la Organización para el Desarrollo y Cooperación Económica (OCDE, 2005) y otros informes internacionales como el Informe McKinsey (Barber y Mourshed, 2007) referidas a "Atraer, Desarrollar y Retener Profesores Efectivos". Estas políticas se examinan a la luz de los énfasis principales de apoyo, regulación, incentivos y control, enmarcados en los propósitos amplios de aseguramiento de la calidad y de rendición de cuentas. El artículo hace notar que los marcos de política estudiados cobran sentido en el contexto de los fenómenos de globalización y de internacionalización de las políticas, pero también en el marco de la ideología neo-liberal y conceptos de mercado presentes en las políticas e instituciones educativas en Chile desde los años ochenta.
\end{abstract}

Palabras Clave: formación inicial docente, gestión de políticas, influencias internacionales.

\section{ABSTRACT}

The article examines Chilean national policies affecting teacher education and its effectiveness over the past fifteen year. The article uses as analytical categories those formulated by OECD (2004) and other international reports such as McKinsey (Barber \& Mourshed, 2008) centred on "Attracting, Developing and Retaining Effective Teachers. The policies are considered in the light of their main emphases directed to support, regulate, and provide incentives and control, and their framing within the broad purposes of quality assurance and accountability. The article notes that the policy frames studied make sense within the context of globalization and internationalization of policies, as well within the neo-liberal ideology and market concepts which have been present in Chilean policies and educational institutions in Chile since the 1980s.

Key Words: initial teacher education, policy development, international influences.

\section{RESUMO}

Examinam-se as iniciativas políticas nacionais que têm afetado a formação inicial de professores e sua efetividade nos últimos quinze anos. Para tanto, utilizam-se as categorias formuladas pela Organização para o Desenvolvimento e Cooperação Econômica (OECD, 2005) e outros relatórios internacionais como o McKinsey Report (Barber \& Mourshed, 2008) que se refere a "atrair, desenvolver e reter professores efetivos". Essas políticas são analisadas à luz da ênfase dada ao apoio, à regulação, aos incentivos e controle, enquadrados nos amplos propósitos da garantia de qualidade e prestação de contas. Observa-se que os quadros políticos estudados fazem sentido no contexto dos fenômenos da globalização e internacionalização da política, mas também no contexto de conceitos da ideologia neoliberal e de mercado presentes nas políticas e instituições educacionais no Chile desde os anos oitenta.

Palavras-chave: formação inicial de profesores, gestão política, influências internacionais.

\footnotetext{
La autora agradece el apoyo recibido para este trabajo del Proyecto FB0003 del Programa de Investigación Asociativa de
} CONICYT. 


\section{INTRODUCCIÓN}

La evolución y características de la formación inicial docente durante los últimos quince años ha sido objeto de diversos estudios centrados en el análisis de las políticas de formación docente (Cox, Meckes y Bascopé, 2010), en su efectividad para producir conocimiento pedagógico general y de contenido matemático (Ávalos y Matus, 2010) y en la relación entre calidad de las instituciones en que se albergan los programas de formación docente y el nivel de conocimiento de los futuros profesores (Pedraja et al., 2012). También se ha examinado la relación entre formación docente y elección del primer empleo y la distribución de nuevos profesores en los establecimientos educativos según tipo de institución formadora de la que egresó (Paredes et al., 2013; Meckes y Bascopé, 2012). Estos estudios y la percepción de quienes siguen de cerca los procesos de formación docente han alertado respecto a una serie de condiciones problemáticas de la formación inicial docente en Chile. Entre estas condiciones se destaca el que la preparación docente no atrae a buenos egresados de la educación media, y que los programas de formación han crecido más en número que en la calidad de las instituciones formadoras, lo que afectaría la competencia de sus egresados. También se hace notar la distribución inequitativa de nuevos profesores en el sistema escolar, en la medida en que quienes supuestamente son mejores profesores por haber sido formados en instituciones más selectivas, no enseñan en los establecimientos escolares que más necesitarían de ellos.

Este clima de preocupación por la calidad de la formación docente explica el desarrollo de una serie de políticas y acciones destinadas a corregir los problemas observados. Así, con el fin de frenar el crecimiento súbito y elevado de la matrícula en las carreras de pedagogía producido por la apertura de programas no convencionales (a distancia o semipresenciales), las universidades agrupadas en el Consejo de Rectores acordaron discontinuar estos programas (Ministerio de Educación de Chile, 2005). Con el fin de monitorear el nivel de conocimientos de los futuros profesores se comenzó a aplicar en el año 2008 una medición de estos conocimientos, conocida como Prueba Inicia. También desde el año 2004 el Ministerio de Educación ha financiado proyectos concursables destinados a mejorar los procesos de formación docente en el marco del programa de Mejoramiento de la Calidad de la Educación Superior (MECESUP), y a partir del 2012 mediante el sistema de Convenios de Desempeño, también concursables. Con la Ley de Aseguramiento de la Calidad de la Educación Superior, promulgada en el año 2006, se decretó la obligatoriedad de la acreditación para todas las carreras de pedagogía. Por último, en el contexto de la preocupación por elevar el nivel de ingreso de estudiantes a las carreras de pedagogía medido por la prueba de selección universitaria (PSU), se estableció una beca especial conocida como Beca Vocación del Profesor.

En lo que sigue de este artículo se examinan estas y otras iniciativas de política nacional que afectan a la formación inicial docente y su efectividad. Para ello se utilizan las categorías formuladas por la Organización para el Desarrollo y Cooperación Económica [OCDE] (2005) y otros informes internacionales como el Informe McKinsey (Barber y Mourshed, 2007) referidas a Atraer, Desarrollar y Retener Profesores Efectivos. A su vez, estas políticas se examinan a la luz de los énfasis principales de apoyo, regulación, incentivos y control, enmarcados en los propósitos amplios de aseguramiento de la calidad y de rendición de cuentas. Se hace notar en el artículo que los marcos de política estudiados cobran sentido en el contexto de los fenómenos de globalización y de internacionalización 
de las políticas, pero también en el marco de la ideología neo-liberal fuertemente presente en las políticas e instituciones educativas en Chile desde los años ochenta.

\section{LA FORMACIÓN INICIAL DOCENTE BAJO LA LUPA}

La era de pruebas estandarizadas nacionales e internacionales referidas al aprendizaje escolar en los sistemas educacionales ha dirigido la atención al rol que juegan los profesores y profesoras en estos resultados, cualquiera sea el nivel de su calidad. Este análisis ha comprendido también a las instituciones de formación por el efecto que pueden tener sus egresados en la calidad del ejercicio docente en las escuelas. Así se ha ido extendiendo la noción en los países que no logran resultados esperados, por ejemplo en la prueba PISA (Programme for International Student Assessment, OCDE), pues la formación docente sería un factor contribuyente a estos resultados y requeriría, por tanto, de seria revisión, como sucedió en Irlanda ante los resultados de PISA 2009 (Meyer, 2013; Conway, 2013). Ante esta situación, las medidas de mejoramiento sugeridas toman la forma de acciones de mayor control y regulación desde el Estado o de acciones que desdibujan el rol de la formación institucionalizada, al favorecer o introducir modos alternativos de reclutamiento para el ejercicio docente. Tres países ejemplifican este tipo de políticas: Nueva Zelanda, Estados Unidos e Inglaterra.

En el caso de Nueva Zelanda, a pesar de tener, en general, resultados satisfactorios en la prueba PISA 2009, el gobierno del país consideró preocupante, respecto a esa prueba, la brecha entre los grupos de "alto" y de "bajo" rendimiento", la más alta en los países OCDE, y expresada en los logros comparativos de la población de origen europeo respecto a los de la población indígena (Maorí y Pacifika). Esto preocupó no sólo en término de valores como la equidad y justicia social, sino que por sus posibles repercusiones en el desarrollo económico del país (Ell y Grundnoff, 2013; O’Neill, 2012). Por ello, y a pesar de existir una preparación docente preocupada por la calidad y la atención a las diferencias culturales del país, comenzó a tomar forma un discurso analítico-crítico respecto al valor que agregaban o no agregaban las instituciones formadoras al aprendizaje escolar, medido por pruebas estandarizadas (Ell y Grudnoff, 2013). Para atender a esta situación, se ampliaron las facultades del gobierno para regular y controlar a la formación docente durante los procesos de acreditación y re-acreditación de los programas de formación (New Zealand Teachers Council [NZTC], 2010). Se acordaron nuevas regulaciones que exigen a las instituciones formadores realizar entrevistas específicas a los candidatos y aplicarles pruebas de habilidades básicas (regulación al ingreso) y mejorar las experiencias de práctica profesional, no permitiéndose más de dos veces la repetición de ésta (regulación al egreso). Sin embargo, a pesar de reconocer la importancia de atender al mejoramiento de la calidad de quienes entran a la profesión y de los que egresan, lo preciso de las regulaciones es que han sido consideradas como una intromisión en la autonomía universitaria y en la capacidad de innovar de las instituciones formadoras (Ell y Grudnoff, 2013). Por su parte, la falta de evidencia concreta aportada por las mismas instituciones formadoras respecto a la efectividad de su trabajo ha contribuido a que se difunda la percepción de que estas instituciones son a la vez "causa" y "solución" del problema definido como rendimiento por debajo de lo requerido del sistema escolar (Ell y Grudnoff, 2013).

Aunque con variaciones en cada uno de los dos países, las políticas dirigidas a docentes en Estados Unidos e Inglaterra tienen elementos en común, en la medida en que 
se fundamentan también en una crítica fuerte a la formación inicial y a la capacidad de sus instituciones de preparar profesores que contribuyan a mejorar el rendimiento de los alumnos. Según los analistas, estas políticas se han orientado por principios neo-liberales que favorecen un amplio mercado de opciones de formación docente y que promueven una intervención estatal de apoyo a este mercado (Furlong, 2006, 2013; Zeichner, 2010). Una de las formas comunes a ambos países y caracterizada por Zeichner (2010) como "desprofesionalización de la enseñanza", descansa en la convicción de que no es necesario adquirir preparación profesional específica para ejercer y que basta simplemente con obtener un grado universitario. A la luz de esta visión, ambos países propician la introducción de alternativas a la formación docente institucionalizada ${ }^{1}$. La declaración de uno de los ministros ingleses del actual gobierno, citada por Furlong (2013) ilustra este bajo nivel de reconocimiento al rol de la formación inicial docente: "Prefiero tener en la escuela a un graduado de Oxbridge sin formación docente, que al graduado de una de las universidades basura que sí tiene formación docente".

Otra de las formas de desprestigio de la formación profesional universitaria, también común a Estados Unidos e Inglaterra, es la que disminuye el rol de la preparación pedagógica propiamente tal, señalando que lo que se necesita saber para enseñar se aprende mejor en los establecimientos escolares. Así, por ejemplo, en Inglaterra se le ha conferido el status de institución formadora a establecimientos escolares selectos, ofreciéndolos como una ruta legal para adquirir la habilitación docente (School-centred Initial Teacher Training, SCITT $)^{2}$. Desde otra perspectiva, estos sistemas educativos han procurado aumentar el control sobre el contenido de la formación docente mediante prescripción sobre su currículo y el establecimiento de estándares precisos referidos a lo que debe saber y ser capaz de realizar el futuro docente. En el caso de Inglaterra, fueron los gobiernos laboristas los que institucionalizaron primero un currículo nacional para la formación docente, reemplazándolo más tarde por estándares genéricos acompañados de orientaciones que vinculan los estándares con políticas referidas al trabajo escolar (Furlong, 2013). En el caso de Estados Unidos existe una larga experiencia de contar con estándares genéricos y específicos que han servido para orientar los procesos de acreditación de las instituciones formadoras.

Respecto al fondo de las críticas a la formación inicial docente en Estados Unidos, Ginsberg y Kingston (2014) argumentan que las más recientes se han construido sobre prácticas que hace tiempo ya fueron modificadas por las propias instituciones, y sobre evidencia discutible como aquella proporcionada por un reciente estudio de los programas de formación docente. Este estudio descalifica irónicamente a los programas de formación por preferir preparar "profesores reflexivos" en lugar de buenos "prácticos", y que habrían sustituido el rol de "entrenar" (training) por la finalidad de "preparar" profesores (National Council on Teacher Quality [NCTQ], 2013)3. En realidad, los sistemas de acreditación en Estados Unidos se han modificado y formado un sistema único más exigente. La evidencia

\footnotetext{
Se refiere específicamente al conocido programa "Teach for All" de gran presencia en Estados Unidos ("Teach for America"), en Inglaterra ("Teach First") y en varios países latinoamericanos incluyendo a Chile y Argentina.

Ver http://www.education.gov.uk/get-into-teaching/teacher-training-options/school-based-training.aspx

"Muchas veces, a lo largo del proceso de Revisión de la Formación Docente nos preguntamos qué podría explicar el carácter caótico del campo de formación docente. Francamente, nuestras primeras teorías eran equivocadas, y fue sólo más tarde durante el proceso en que tropezamos con la evidencia de que el campo [la formación docente] había decido que su función no era entrenar profesores sino que formarlos. Aunque estos dos términos "formar" y "entrenar" parecen intercambiables, en realidad, no lo son". (NCTQ, 2013: 93).
} 
aportada a partir de datos que recoge la asociación nacional de formación docente (AACTE) indica también que las prácticas docentes se han fortalecido en cantidad y calidad y se destaca la reciente iniciativa de evaluación de la capacidad de desempeño docente mediante un portafolio que es usado en 24 estados como criterio para la habilitación docente. Permanece, sí como elemento discutible, la gran presión en Estados Unidos por evaluar la formación inicial docente sobre la base del valor que agregan sus egresados al aprendizaje de los alumnos medido en pruebas estandarizadas, fuente a su vez de potenciales demandas de "rendición de cuentas" a las instituciones formadoras (ver Glazerman et al, 2010).

La formación docente en Chile ha sido objeto de atención crítica por parte de las autoridades educacionales de los gobiernos democráticos con distinta intensidad y focos de atención desde mediados de los años noventa hasta hoy. Esta atención ha estado invariablemente asociada a las necesidades de mayor escala del sistema educativo referidas a mejorar los resultados de aprendizaje, medidos por las pruebas nacionales SIMCE (Sistema de Medición de la Calidad de la Educación). El primer llamado de atención respecto al rol de los docentes y de su formación respecto al mejoramiento de los procesos educativos, ocurrió a mediados de los años noventa con el establecimiento del programa de Fortalecimiento de la Formación Inicial Docente (FFID). Este programa estuvo destinado a estimular procesos de cambio en una formación docente muy debilitada durante los años de dictadura, tanto en sus recursos académicos como en el número reducido y baja calidad de los interesados en ingresar a las carreras de pedagogía. El programa FFID (1997-2002) representó una acción decidida del Estado mediante el otorgamiento de recursos económicos en forma competitiva a las instituciones dispuestas a comprometerse en procesos de mejoramiento. Junto con el uso de estos mecanismos competitivos o de mercado, el programa contempló un fuerte sistema de apoyo desde el Ministerio de Educación en la implementación de los proyectos preparados por las universidades participantes, y favoreció el trabajo conjunto de las instituciones como medio de monitoreo y aprendizaje (Ávalos, 2002). El hecho de que beneficiara a alrededor del $80 \%$ de los estudiantes de pedagogía de la época, constituyó una señal clara por parte del Estado en el sentido de apoyar la reforma en la mayor parte de las instituciones viables o capaces de auto-reformarse. El programa FFID logró efectos importantes en cuanto al aumento del número de estudiantes de pedagogía, como lo muestra la Figura 1, y en los puntajes promedio (PAA), los cambios mayores ocurrieron en las carreras de Educación Básica (de 540 a 587 puntos) y en las carreras de Educación Media (de 533 a 589).

Figura 1. Evolución de la matrícula total en carreras de pedagogía de 17 universidades participantes del programa FFID

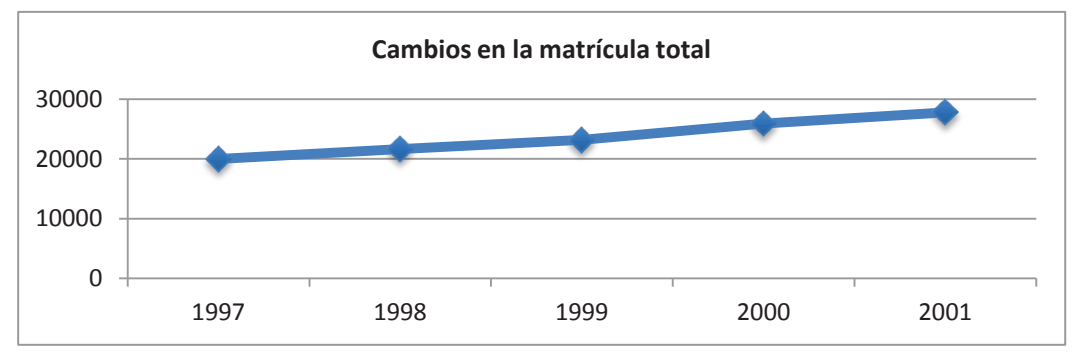

Fuente. Ávalos (2002). 
Cumplida esta etapa, se resolvió no continuar el apoyo a las instituciones FFID que, si bien había progresado bastante con respecto a su situación inicial, necesitaban específicamente de mayor avance en los contenidos curriculares de la formación y afianzar un sistema de autoevaluación mediante estándares orientadores (Ávalos, 2002; Cox, Meckes y Bascopé, 2010). En esta situación de inercia por parte de las autoridades de gobierno y casi sin advertirlo, comienza a producirse a partir del año 2002 un aumento explosivo en la oferta de nuevas carreras de pedagogía por parte de universidades privadas y también de algunas estatales, reflejadas en programas a distancia o semi-presenciales y en apertura de sedes a lo largo del país con poca capacidad para ofrecer buenos procesos de formación. La matrícula total en carreras de pedagogía de universidades privadas entre los años 2000 y 2008 registró aumentos de $812,6 \%$ en las carreras de educación básica y de $940,7 \%$ en las carreras de educación media, esto comparado con aumentos de 174,4\% y 74,7\% respectivamente en las universidades tradicionales (Cox, Meckes y Bascopé, 2010). Es decir, cualquier elemento de mejora ocurrido durante el programa FFID no podía afectar a esta enorme nueva oferta surgida en el período señalado.

Sólo con la introducción de la acreditación voluntaria para las carreras de pedagogía en el 2003 y la decisión en 2005 adoptada en conjunto por el Ministerio de Educación y el consorcio de universidades tradicionales CRUCH (Consejo de Rectores de las Universidades de Chile) de cerrar los programas a distancia (Ministerio de Educación de Chile, 2005), se advierte el comienzo tímido de una acción reguladora de la formación docente por parte del Estado, en que se conjugan los esfuerzos por mejorar la formación inicial docente mediante instrumentos competitivos de mercado con políticas de incentivos y control sobre ella.

\section{LAS DEMANDAS SOCIALES Y LAS NUEVAS POLÍTICAS DE FORMACIÓN DOCENTE (2006-2014)}

El movimiento social de jóvenes conocido como de "los pingüinos", que irrumpió en el escenario chileno el año 2006 reclamando por una mejor educación pública, tuvo como efecto la constitución de una comisión representativa de todos los sectores preocupados por la educación para diagnosticar la situación educativa y proponer políticas de cambio (Consejo Asesor Presidencial para la Calidad de la Educación). Uno de los temas centrales de deliberación durante el período en que sesionó el Consejo Asesor fue el de la formación inicial docente. Entre los principales asuntos que incluyó su diagnóstico estuvo el reconocimiento de problemas derivados de los programas mismos, como la calidad y relevancia del currículo para las necesidades del sistema escolar, incluyendo la educación especial y la educación técnico-profesional, y problemas derivados de la apertura no regulada de nuevas carreras de pedagogía como también la falta de precisión acerca de las consecuencias para una institución formadora de fallar en sus intentos de acreditación. Entre las propuestas más importantes para monitorear, regular, y promover la calidad de la formación inicial docente se propuso el establecimiento de una "institución reguladora que contribuya a conectar de manera coherente y efectiva las actividades de formación docente inicial y continua y sentar bases para su desarrollo futuro en el país" (Consejo Asesor Presidencial, 2006). Esta institución debía encargarse de: (a) formular políticas orientadoras para el desarrollo de la formación inicial docente y continua; (b) autorizar la apertura de 
nuevas carreras de pedagogía velando por que cumplan con los estándares requeridos para una formación docente de calidad; (c) acreditar las carreras de pedagogía según lo establecido por el Sistema de Aseguramiento de la Calidad de la Educación Superior; (d) establecer las exigencias de entrada al ejercicio de la profesión docente (habilitación) y determinar los apoyos necesarios durante el comienzo de su ejercicio (inducción); y (f) actuar como un observatorio de la calidad de la formación docente, evaluando sus programas e informando sobre sus resultados (acreditación, habilitación y otros indicadores), y realizar o encargar estudios referidos al desarrollo y mejora de la formación docente. Además de esta propuesta, se recomendó en forma específica la realización de un examen de habilitación para los futuros profesores antes de su egreso de la formación docente. El examen se centraría en conocimientos disciplinarios y de pedagogía y los resultados determinarían la elegibilidad para ser contratado en el sistema público de educación.

Los lineamientos de política diseñados por el Consejo Asesor, y en particular el establecimiento de una institucionalidad regulatoria y de apoyo de las instituciones formadoras, hubiesen significado un avance importante hacia un sistema de formación inicial docente de calidad. Lamentablemente, la única propuesta implementada más tarde, aunque no aprobada en su momento por el Consejo Asesor, fue el examen de conocimientos disciplinarios y pedagógicos, pero sin carácter de habilitante. Esto significó que en los años siguientes se empezaron a configurar políticas en forma parcelada respondiendo a situaciones críticas a medida que ellas se iban presentando. Esta configuración de políticas, si bien emergente en los últimos gobiernos, ha respondido a criterios que son asimilables a los encabezamientos de política internacional docente (Barber y Mourshed, 2007; OCDE, 2005) y a lógicas tensionadas entre apoyo y aseguramiento de la calidad vía presión y control. La figura siguiente ilustra en forma sintética tanto los marcos interpretativos usados en este artículo como la naturaleza de las principales políticas y acciones realizadas en el período 2008-2014.

Figura 2. Políticas y acciones dirigidas a la Formación Docente Inicial (2008-2014)
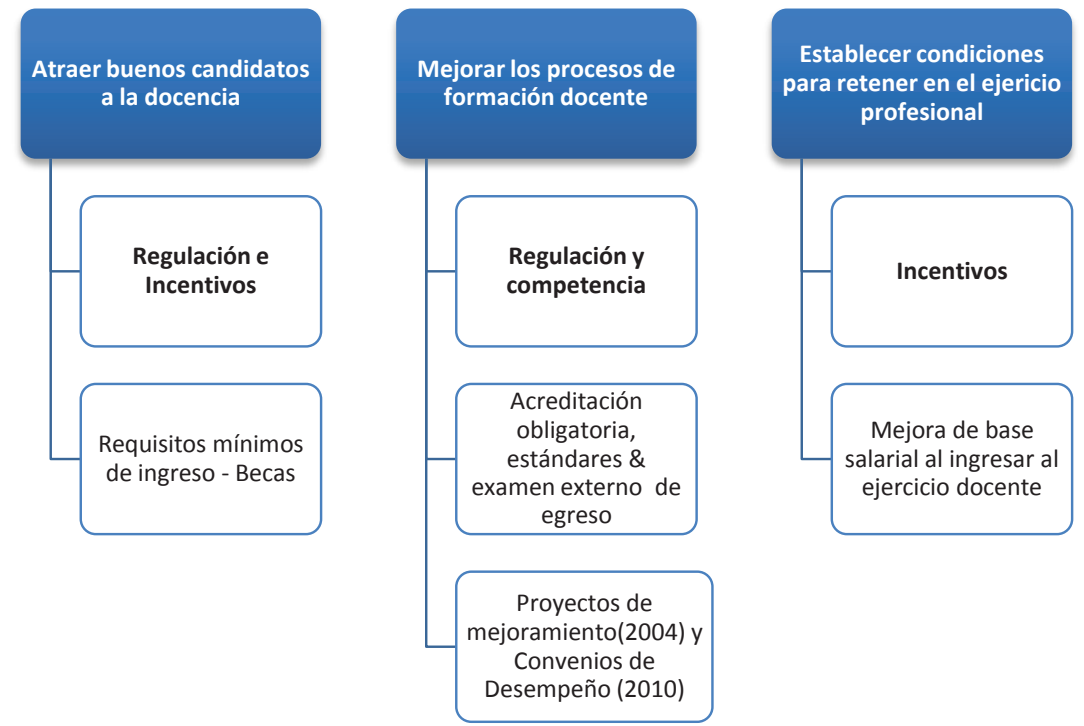

Fuente. Elaboración propia. 


\subsection{ATRAER MEJORES CANDIDATOS AL EJERCICIO DOCENTE}

Si bien los puntajes de ingreso a la formación docente mejoraron hacia el fin del programa FFID como se señaló en la sección 1, el enorme aumento de oferta de formación docente de las instituciones privadas redujo el promedio de estos puntajes, pero en forma diferenciada por tipo de institución. La Tabla 1 muestra el promedio general de puntajes de admisión de 500 puntos o más en el período 2005 a 2011. Como se observa, la proporción de jóvenes que ingresó a las carreras de pedagogía con puntajes de 500 puntos o más es mucho mejor en las universidades CRUCH que en las privadas. Los Institutos Profesionales, que también ofrecen carreras de pedagogías, no requieren el rendimiento de la PSU, aunque algunos sí lo hacen.

Tabla 1. Promedio de estudiantes con puntajes PSU de 500 o más puntos admitidos en las Carreras de Pedagogía por tipo de institución (años 2005-2011)

\begin{tabular}{|cccc|}
\hline Tipo de Universidad & Nivel Parvulario & Nivel Básico & Nivel Medio \\
\hline CRUCH & $89 \%$ & $100 \%$ & $100 \%$ \\
Privadas & $28 \%$ & $36 \%$ & $41 \%$ \\
\hline
\end{tabular}

Fuente. CEPPE, Notas para Educación, Septiembre 2012.

La intención de mejorar los niveles de ingreso a las carreras de Pedagogía se había manifestado ya en la Beca para Profesores que formó parte del programa FFID y que continuó ofreciéndose después del término de este Programa. Esta beca contribuyó a aumentar los niveles de ingreso en alrededor de un 7\% a 9\% según lo indica el único estudio sobre el programa (Toledo, 2009). Sin embargo, la cobertura de esa beca era reducida y se requería de mejores incentivos para estimular el ingreso de buenos candidatos a las carreras de pedagogía. Esto ocurrió con el establecimiento de la llamada "Beca Vocación del Profesor" otorgada por primera vez en el año 2010 sobre la base de un puntaje mínimo de ingreso de 600 puntos y el requerimiento de que la carrera pedagógica a la que se accedía fuese acreditada y tuviese como requisito de ingreso un puntaje mínimo de 500 puntos. Adicionalmente, la Beca ofreció mayores incentivos a quienes ingresaran con puntajes mayores que el mínimo exigido (mejores aportes monetarios y un semestre de estudios en el extranjero). Al igual que con la beca anterior, se estableció que los nuevos profesores beneficiados con esta beca debían ejercer por tres años en el sector escolar subvencionado. Los efectos de la Beca fueron inmediatos en términos de aumentar la proporción de quienes ingresaron a las carreras de pedagogía (54\% tuvo puntajes de 550 puntos o más). Sin embargo, en las recientes admisiones (2013 y 2014) se muestran signos de estancamiento. Es así como el año 2013 hubo un 11\% de menos becados que el 2012 (La Tercera, en línea) y esto ha ido aparejado con un descenso general en la matrícula de las carreras de pedagogía, como lo muestra la Figura 3. Este descenso, que puede atribuirse a problemas de salario y de relación tiempo lectivo/no lectivo de los docentes, sugiere que para atraer mejores candidatos a la docencia no es suficiente el proporcionar becas o incentivos monetarios atrayentes, sino que es necesario mejorar las condiciones de ejercicio de los docentes. 
Figura 3. Matrícula total carreras de pedagogía

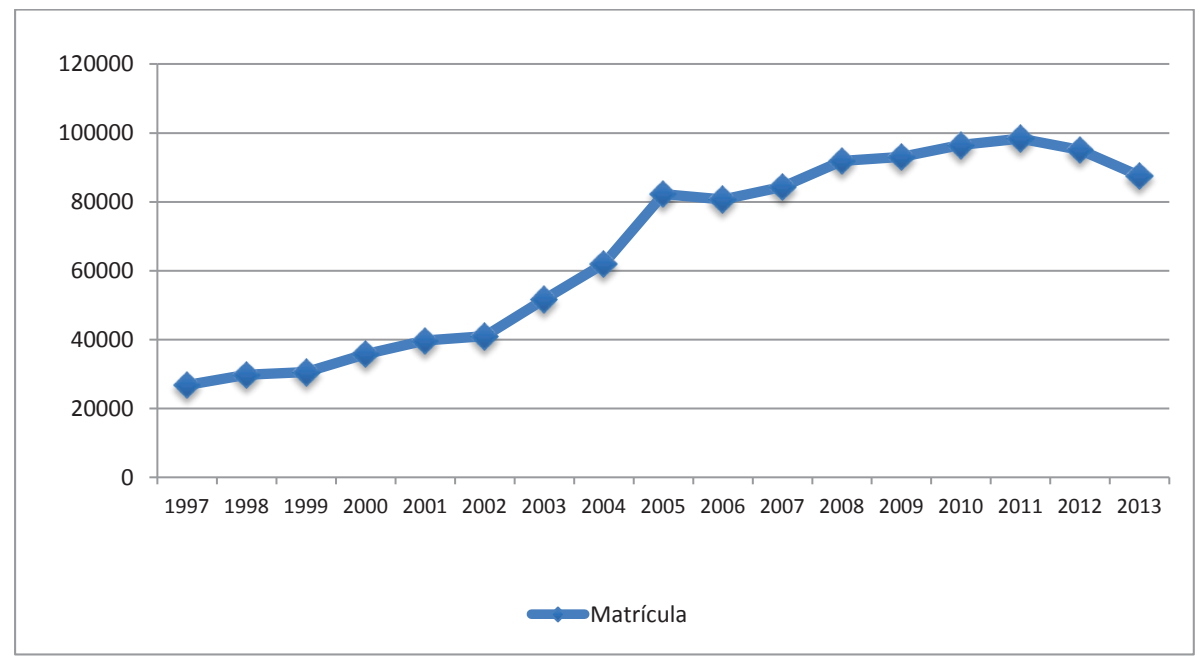

Fuente. Consejo Nacional de Educación.

\subsection{EL MEJORAMIENTO DE LOS PROCESOS FORMATIVOS}

La forma de influir en el mejoramiento de la "caja negra" de los procesos formativos (el currículum y las estrategias docentes) en la era posterior al programa FFID ha tomado dos formas principales: (a) procesos de aseguramiento de la calidad; y (b) financiamiento competitivo de proyectos de cambio mediante la fórmula de "convenios de desempeño".

\subsubsection{El aseguramiento de la calidad}

El principal mecanismo utilizado para este fin es la acreditación obligatoria de las carreras de pedagogía. Este proceso comenzó en forma voluntaria bajo el alero del Consejo Nacional de Acreditación de Pre-Grado (CNAP) en el año 2004, pero se estableció su obligatoriedad para las carreras de pedagogía y medicina en la Ley de Aseguramiento de la Calidad de la Educación Superior (2006). En su formato original se incluyó como criterios para la evaluación de procesos a los estándares genéricos producidos por el proyecto FFID y consensuado con los programas de formación inicial docente en 2002. En su forma actual, si bien los criterios son similares, los procesos mismos de acreditación han sido entregados a agencias privadas contratadas por las instituciones que requieren acreditación de sus programas, aun cuando su resultado final es decidido por la Comisión Nacional de Acreditación. En la práctica aunque la mayoría de los programas de formación docente se ha ido sometiendo a los procesos de acreditación, hasta el 2012 no todos habían sido cubiertos, era el caso de pedagogías en Educación Básica, Pedagogía en Castellano y Pedagogía para Licenciados (ver Tabla 2). Hay diversas críticas a los procesos de acreditación actuales. Se considera que su impacto es limitado en lo que se refiere a cambiar los procesos formativos mismos (Cox, Meckes y Bascopé, 2010). Se ha objetado además la privatización de los procesos de acreditación de programas como también su efectividad para verificar la calidad misma de los programas de formación (Panel de Expertos, 2010). Esto, en parte, porque la 
acreditación se puede otorgar por períodos menores al máximo de siete años establecido, porque la selección de agencias está en manos de quienes necesitan ser evaluados y porque ciertas carreras sólo logran acreditación por períodos cortos de uno a tres años. Respecto a esto último, sin embargo, un estudio que examinó el grado de asociación entre años de acreditación y resultados en el examen de egreso de las pedagogías no encontró una relación significativa entre estos dos factores (Domínguez et al., 2012)

Tabla 2. Estado de Acreditación de Programas de Formación Docente

\begin{tabular}{lccc}
\hline Programas & $\begin{array}{c}\mathrm{N}^{\circ} \text { programas } \\
\text { vigentes }\end{array}$ & $\begin{array}{c}\mathrm{N}^{\circ} \text { programas } \\
\text { acreditados vigentes }\end{array}$ & \% acreditación \\
\hline Educación Parvularia & 141 & 108 & $77 \%$ \\
Educación Básica con/sin menciones & 141 & 92 & $65 \%$ \\
Educación Diferencial & 67 & 49 & $73 \%$ \\
Pedagogía Media para licenciados & 21 & 10 & $63 \%$ \\
Educación Media & & & \\
- Inglés y similares & 93 & 79 & $85 \%$ \\
- Educación Física & 88 & 73 & $83 \%$ \\
- Historia y Geografía & 57 & 43 & $75 \%$ \\
- Matemáticas & 43 & 32 & $74 \%$ \\
- Castellano y similares & 38 & 24 & $73 \%$ \\
- Biología, Química y similares & 30 & 22 & \\
\hline
\end{tabular}

Fuente. Consejo Nacional de Educación, Programas vigentes de instituciones autónomas (universidades, institutos profesionales y centros de formación técnica), Noviembre 2012.

El segundo mecanismo para asegurar calidad ha sido la confección de estándares "orientadores" para las carreras de pedagogía, que a su vez debieran alinearse con el examen de conocimientos que se aplica al término de los estudios de pedagogía. Estos estándares son principalmente de contenido disciplinar y contenido pedagógico, aunque incluyen también referentes didácticos para el contenido disciplinar. Sin embargo, si bien estos estándares se discutieron durante el proceso de su elaboración con las instituciones formadoras, no han tenido la posibilidad aún de orientar la adecuación de los currículos de formación docente, pues sólo fueron entregados en su forma final a comienzos del año 2013.

El tercer mecanismo de aseguramiento de la calidad, pero más específicamente de control sobre la formación inicial docente, ha sido el examen de egreso (Prueba INICIA) que se ha mencionado antes. Este examen es aplicado desde el año 2008 en forma voluntaria, aunque con la intención de que se convierta en obligatorio y que tenga algún tipo de consecuencia para el posterior ejercicio docente. En este sentido, un proyecto de ley enviado al Congreso en 2010, modificado el 2013 pero finalmente retirado de discusión, proponía que el examen fuese condición de empleo en el sector público y particular subvencionado, aunque sin tener un carácter habilitante. Es decir, el examen no tendría más efecto que condicionar la postulación a un establecimiento educacional, pero sin establecer exigencias mínimas acerca de la calidad del rendimiento en esa prueba. 
Respecto al momento de aplicación del examen, sus contenidos y la publicidad dada a sus resultados, hay opiniones críticas serias. El examen, conocido como INICIA, se ha estado aplicando al egreso de los futuros profesores. Sin embargo, como lo indica GarcíaHuidobro (2011) no es oportuno aplicar una prueba cuando los futuros profesores están egresando y hay pocas posibilidades de mejorar aquello que resulta insatisfactorio en su rendimiento. Sería mejor hacerlo un año antes del egreso, cuando se ha completado el trabajo de cursos y está por comenzar el período de práctica profesional. Respecto a los contenidos del examen, sólo en la última aplicación a los egresados en el 2012, estos estuvieron alineados con los estándares orientadores pero, a su vez, un grupo de esos estándares sólo fue recibido por las instituciones formadoras en ese mismo año y, por tanto, es posible que no hubiesen tenido buena relación con el currículo enseñado en las instituciones formadoras. Es decir, en sus diversas aplicaciones hasta el 2012, la prueba podría carecer de validez debido a la inadecuada relación con los contenidos de la formación docente cuyo aprendizaje se desea medir. Si se han de evitar los efectos no intencionados y promover aquellos intencionados, como mejorar los procesos de formación, es necesario prestar atención a la validez (Haertel, 2013). También en relación a los contenidos, la forma del examen no permite evaluar la capacidad de enseñanza y gestión en las aulas (GarcíaHuidobro, 2011), y por tanto no puede asegurar que se poseen las mínimas condiciones para el ejercicio docente, como lo expresó muy bien en una carta al periódico El Mercurio una de las futuras profesoras evaluadas en el año 2013:

\section{Señor director:}

Soy egresada de Pedagogía de una universidad del Consejo de Rectores. Ponderé más de 600 puntos en la PSU y mis resultados en la Prueba Inicia estuvieron dentro del primer quintil de quienes rendimos ese año. Pese a ello, no podría afirmar que me encuentro preparada para ejercer la labor docente, trabajo complejo en el que intervienen múltiples factores que no son susceptibles de ser medidos por una evaluación estandarizada. No se puede asegurar el carácter predictivo de la prueba.

La lógica de medir todo y premiar o castigar de acuerdo al desempeño es nefasta para el sistema educativo, que segrega y no contribuye a mejorar la educación.

Por otra parte, la prueba sólo mide conocimientos específicos en algunas áreas, aunque un docente no es alguien que transmite conocimiento, sino alguien que media para la construcción del mismo. Por lo anterior, no es necesario que tenga su cabeza llena de información: la pedagogía es mucho más compleja que eso.

Las condiciones mínimas y dignas en las que deben trabajar los profesores son una obligación del Estado y no deben considerarse como un premio al desempeño. Mejorar la formación inicial docente es una responsabilidad compartida y no individual; por cada docente con mala formación detrás hay una institución que no hizo bien su trabajo, y un Estado que falló en su rol fiscalizador.

Paulina

Finalmente, a pesar de la intención inicial de no hacer públicos los resultados del examen esto no ha ocurrido en sus últimas aplicaciones, lo que además de no contribuir a mejorar los procesos formativos tiene, por lo menos, dos efectos no deseados: que en el futuro las instituciones realicen actividades de adiestramiento para el examen y, más grave aún, que puedan influir en las decisiones de contratación de nuevos profesores sobre la base de la institución en que estudiaron, sin considerar si el postulante tiene o no las capacidades requeridas para el empleo. 


\subsubsection{Mejoramiento de los procesos de formación mediante financiamiento competitivo}

El concepto de inducir mejoramiento en los procesos de formación mediante mecanismos competitivos fue parte del programa FFID al que se hizo mención en la primera parte de este artículo. Dos años después de terminar ese programa, el Ministerio de Educación, a través del programa de Mejoramiento de la Calidad de la Educación Superior (MECESUP), ofreció fondos competitivos para la realización de proyectos orientados a modificar los currículos de formación para la Educación Básica mediante la introducción de especializaciones o menciones referidas a las principales disciplinas del currículo escolar (Lenguaje, Matemáticas, Ciencias Sociales y Ciencias Naturales). A su vez, el diseño y ejecución de estos proyectos se condicionó al establecimiento de consorcios de universidades y al uso del sistema de competencias del Proyecto Tuning de la Unión Europea. A diferencia del modo de operar del programa FFID, estos proyectos MECESUP no recibieron apoyo directo del Ministerio de Educación, salvo en lo que respecta al monitoreo de su ejecución.

Si bien la mayoría de los programas de formación docente involucrados en proyectos MECESUP realizaron cambios a su currículo de formación según lo requerido, nunca hubo un cierre adecuado de los proyectos ni una evaluación de su efecto en el tiempo (Cox, Meckes y Bascopé, 2010). Por el contrario, se produjo una situación compleja para las instituciones que introdujeron las especializaciones curriculares indicadas debido a la modificación del sistema escolar establecido por la Ley General de Educación (2008). Esta modificación, prevista para el año 2018, establece un sistema de seis años de educación básica y seis años de nivel secundario. En relación a este cambio, el Ministerio de Educación dio señales respecto a la necesidad de preparar profesores generalistas en cuatro menciones para los seis años de Educación Básica, en lugar del énfasis anterior de preparación general y de preparación en una o dos menciones disciplinares. Esto se ha reflejado en la construcción de la prueba INICIA que examina en cuatro áreas curriculares sin considerar la situación de quienes han sido preparados con mención disciplinar (García-Huidobro, 2011). Dicho de otro modo, el mejoramiento del currículo de formación de profesores de Educación Básica ha sido objeto de políticas contradictorias que han complicado los procesos de formación y, por lo mismo, el mejoramiento de su calidad.

Más recientemente, los programas de financiamiento competitivo se han reconvertido en un sistema de "Convenios de Desempeño" con dos convocatorias en los años 2012 y 2014. Lo central de estos convenios es estimular la realización de "análisis estratégico y cambio institucional” (Ministerio de Educación, 2014) con el fin de preparar profesores que posean un alto nivel de competencias. Esto, a su vez, debiera demostrarse en mayor aprendizaje en las aulas de los y las profesoras egresadas de las instituciones favorecidas. Estos convenios se definen formalmente como:

(...) un instrumento competitivo de adjudicación de recursos de avanzada categorías, de alto impacto estratégico que provee de financiamiento en base a resultados y permite a la institución generar iniciativas transformadoras, capaces de enfrentar problemas estructurales de diferente tipo y focalizar sus esfuerzos institucionales en aspectos asociados al mejoramiento de la calidad de su quehacer. (Ministerio de Educación, 2014)

Como se observa, el foco de los Convenios de Desempeño está en el logro de resultados cuantificables que comprometan fuertemente a las instituciones a generar acciones conducentes a los resultados esperados. Para ello se especifican dos tipos de indicadores: 
de resultado, entregados por cualquiera sea el examen vigente al momento del egreso de futuros profesores (INICIA u otro) e indicadores de impacto. Estos últimos se definen como el valor agregado por los nuevos profesores al aprendizaje de sus alumnos, y ciertamente, constituyen el indicador más complejo de medir, dadas las grandes dificultades técnicas de hacerlo (Glazerman et al., 2010).

Además de los dos indicadores centrales señalados arriba, los Convenios de Desempeño incluyen otros cinco objetivos precisos que debiesen lograr los proyectos, y suponen que su realización es de entera responsabilidad de la institución que recibe el financiamiento, sin otro tipo de apoyo o de consideración de los contextos distintos en que pudieran implementarse. En términos generales, esta forma de apoyo financiero claramente se aparta de proyectos que buscan innovar o centrarse en aspectos de la formación que no son fácilmente verificables mediante los dos tipos de indicadores señalados. Pero, aún más preocupante es que tanto el indicador de resultados como el de impacto presentan problemas. Por una parte, la prueba INICIA, hasta el momento, no es una prueba que cumpla con todo lo requerido por los criterios de validez y confiabilidad como se ha indicado antes, y por tanto, no es al momento un buen indicador de la efectividad de los Convenios. Por otra parte, la medición de impacto a través del valor que agregan los nuevos profesores al aprendizaje de sus alumnos y alumnas es técnicamente complejo, y no aconsejable como medio de "rendición de cuentas", como lo indican dos revisiones de literatura sobre su efectividad para este fin (Berliner, 2014; Konstantopoulos, 2014).

\subsection{RETENCIÓN DE BUENOS PROFESORES EN EL SISTEMA EDUCATIVO}

Sin ninguna duda, todos los esfuerzos realizados por atraer buenos candidatos a la profesión docente y formarlos en la mejor forma posible, no traerán frutos si quienes egresan no se emplean en el sistema educativo, especialmente en aquellos establecimientos donde más se los necesita, o no permanecen en el ejercicio por un tiempo razonable. Existe evidencia de tasas altas de abandono de docentes en los primeros años de servicio, de alrededor de un 40\% (Valenzuela y Sevilla, 2012). Aun cuando no existe suficiente información sobre sus razones, es posible que este abandono tenga que ver con las condiciones y contextos de trabajo como la cantidad de alumnos y alumnas que deben atender y el elevado número de horas lectivas frente al tiempo disponible para preparar clases y realizar otras tareas relacionadas con su trabajo como docentes. Esto deja entrever la necesidad de establecer mejores condiciones de trabajo para los docentes, especialmente para aquellos que comienzan a enseñar, lo que se relaciona con la necesidad de establecer un carrera para docentes que mejore las condiciones actuales de ejercicio.

En lo que se refiere a profesores y profesoras que se inician en la docencia, hubo un intento de legislar en torno a mejores condiciones en el año 2013. Efectivamente, el gobierno del momento envió al Congreso un proyecto de ley proponiendo condiciones especiales de ingreso al ejercicio para nuevos profesores que tuviesen buenos antecedentes en el puntaje de la Prueba de Selección Universitaria con que ingresó a sus estudios, en el promedio de notas durante estos estudios o en sus calificaciones en el examen de egreso (INICIA). El problema de la propuesta es que al otorgarle un salario más alto a este tipo de docente se discriminaba a otros con menores antecedentes, pero igualmente contratados para ejercer las mismas funciones en los establecimientos educacionales. Es decir, en cierta medida el proyecto atentaba contra la equidad al proponer sueldos diferenciados al ingreso, 
sin que ninguno de los dos tipos de profesores se hubiese probado en su capacidad de enseñar y manejar el grupo curso. Esta propuesta contravenía además las Recomendaciones Referidas al Personal Docente (UNESCO, 2008) que señalan que las retribuciones a los docentes deben relacionarse con el tipo de responsabilidades que adquieren cuando comienzan a enseñar (X. 115).

Lo cierto, en lo que se refiere a políticas para estimular la retención de nuevos docentes, es que no hay hasta el momento una propuesta viable, más allá de mucha discusión pública y declaraciones del gobierno actual en torno a la necesidad de contar con un proyecto de carrera docente que mejore las condiciones de ingreso y ofrezca estímulos salariales y de condiciones de ejercicio adecuadas durante todas las etapas del ejercicio profesional. No ha habido tampoco una propuesta viable para apoyar el ingreso de nuevos docentes en relación a las demandas y dificultades que ocurren al comenzar a enseñar; lo que usualmente se conoce como un programa de inducción para profesores nuevos.

\section{DISCUSIÓN Y CONCLUSIONES}

Lo analizado en este artículo respecto al escenario de políticas referidas a la formación inicial docente cobra sentido en el marco del profundo impacto de las políticas neo-liberales heredadas del período de dictadura (1973-1990), presentes en las estructuras educacionales como también en el modo de encarar la formulación de políticas. Esto a pesar de que quienes votaron por retornar a la democracia buscaban el logro de mejoras sociales y, con respecto a la educación, una mejor calidad distribuida equitativamente. Estos dos elementos explican el que haya existido, en este período de veinte años, tensiones entre quienes afirman la importancia de la educación como factor de crecimiento económico y principios de libre competencia y mercado, y quienes reconocen el poder de la educación como palanca para sacar a las personas de la pobreza y desarrollarlos como actores en el logro de una sociedad más equitativa.

Por otra parte, las políticas educacionales en Chile también pueden entenderse en el marco de una sociedad que ha buscado abrirse al mundo, abandonar el aislamiento de la comunidad internacional ocurrido durante la época militar y establecer condiciones de mayor participación en el mundo global. Como señalan Rizvi y Lingvard (2010) toda política educacional debe considerarse a la luz del conjunto político más amplio del que puede ser parte, y que en el caso de Chile explica la adopción de políticas educacionales que permiten mayor interacción a nivel global como la participación en pruebas estandarizadas internacionales, el financiamiento de becas para estudios avanzados en instituciones prestigiosas fuera de Chile y el uso de instrumentos de política educativa considerados como efectivos. Por tanto, como lo veremos enseguida, no es extraño que en el desarrollo de políticas dirigidas a los docentes y su formación se observen situaciones similares a las descritas en la primera parte de este artículo, por ejemplo, en Estados Unidos (Zeichner, 2010) y en Inglaterra (Furlong, 2006, 2013).

Al evaluar en su conjunto el desarrollo de las políticas dirigidas a la formación inicial docente desde mediados de los años noventa, se observa que los temas centrales, los énfasis e instrumentos usados se han movido desde una intención amplia de acompañamiento y soporte financiero observable en el programa FFID, hasta un énfasis circunscrito y centrado en logros precisos a cambio de soporte financiero, es decir a lo que Zeichner (2010) llama 
"empaquetamiento vendible" de la formación inicial docente. Dicho de otro modo, si bien este desarrollo de políticas en todas sus etapas comparte el mismo diagnóstico referido a la insuficiente calidad de la formación docente, el modo de concebir su mejora ha evolucionado desde el uso preferente de instrumentos de corrección del problema de amplia cobertura como la acreditación obligatoria hacia instrumentos acotados de control y aseguramiento de la calidad, como el uso de estímulos financieros basados en los resultados de exámenes. La Figura 4 ilustra esta evolución:

Figura 4. Evolución de políticas dirigidas a la formación inicial docente (1997-2014)

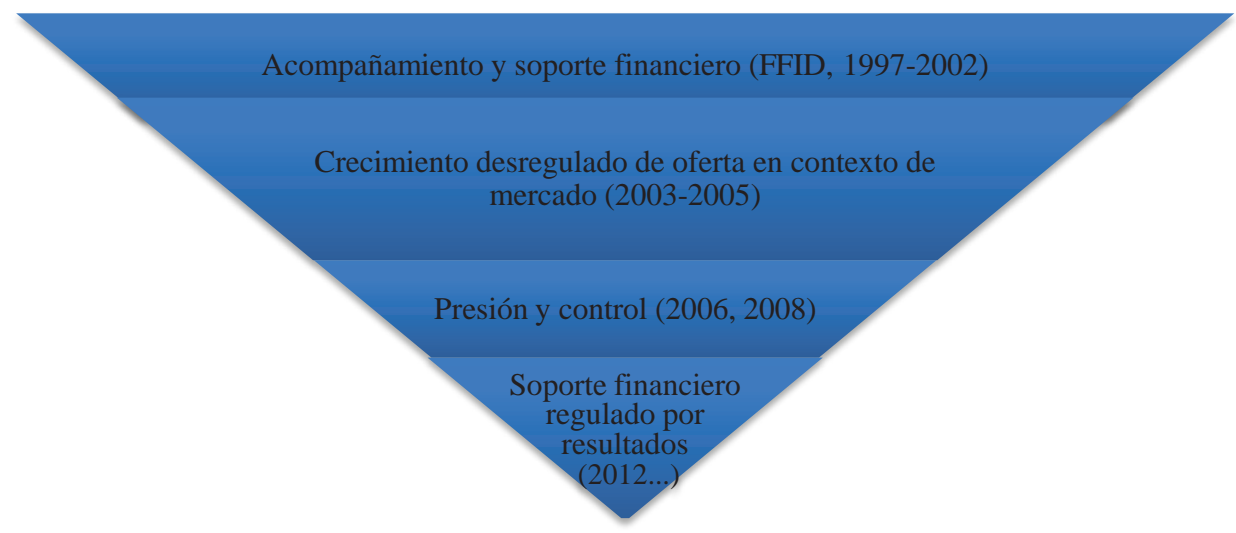

Lo que se busca ilustrar con esta Figura es que la tensión permanente entre apoyo y regulación, que es parte de las políticas de mejoramiento educativo, en el caso de la formación docente en Chile ha significado una creciente disminución del apoyo en los instrumentos de financiamiento para los procesos de mejora. La Figura sugiere que a raíz del crecimiento poco regulado de oferta de formación que comenzó en los años 2000, corregido sólo en las instituciones tradicionales pero no en las privadas, se ha avanzado en el uso de instrumentos de presión y control como el examen basado en estándares (INICIA) y más recientemente en conceder apoyo financiero para la mejoría de los procesos de formación, pero ello circunscrito a modelos específicos y a indicadores cuantitativos problemáticos de resultados e impacto (Convenios de Desempeño). Estas tendencias han operado en forma similar en otros contextos nacionales como se ilustró en la primera parte de este artículo y señalan un camino preocupante hacia la "desprofesionalización" de la docencia. Esto porque en la medida en que el indicador clave para determinar la calidad de un egresado sea el nivel de conocimientos referidos a la disciplina que enseña, tal como lo mide la prueba INICIA, se allana el camino para prescindir de formación docente amplia e institucionalizada y para adoptar modelos de mínima formación. O, desde otra perspectiva, al no contar con instrumentos adecuados para evaluar la capacidad docente de quienes han sido formados en instituciones especializadas, sugerir que bastaría para ejercer que la formación consecutiva de licenciados en alguna disciplina se realice en establecimientos escolares declarados como sedes de formación como ocurre en Inglaterra (Furlong, 2013), o que simplemente se avale el ejercicio mediante una prueba al estilo INICIA, como lo permite la actual Ley General de Educación (2009). 
No se ha llegado en Chile a los extremos señalados por Zeichner (2010) de experiencias dirigidas a desinstitucionalizar la formación docente; pero esto podría ocurrir en la medida en que no se preste atención a los efectos de largo alcance que tendría el uso de ciertos instrumentos de presión que no son examinados en sus interrelaciones, o en sus efectos. Hay buenas señales al momento de valorización de la profesión docente expresadas en un creciente clamor por que se mejoren las condiciones de trabajo de los docentes y se establezca una carrera profesional digna para ellos (Elige Educar, 2012). A la luz de estas señales, es importante examinar en forma integrada las acciones que puedan contribuir a mejorar la calidad de la formación docente. Ello significa advertir que el "atraer" buenos candidatos sólo sobre la base de buenos puntajes de ingreso puede dejar a zonas de Chile, alejadas del centro metropolitano, sin candidatos elegibles (Centro de Estudios de Políticas y Prácticas en Educación [CEPPE ], 2012); significa también que declarar la obligatoriedad de un examen de conocimientos disciplinarios al egreso de la formación docente sin verificar la capacidad práctica para enseñar no asegura la calidad futura del ejercicio docente (García-Huidobro, 2011). Una mirada integrada de políticas y su puesta en acción requiere mirar a la luz del conjunto de ellas sus efectos positivos y negativos y, habiendo decidido cuáles son importantes, verificar si existen condiciones para su aplicación y los plazos en que es posible ponerlas en acción.

El mejoramiento de la formación inicial docente ciertamente requiere de regulación respecto a la cantidad de programas que son viables y necesarios para los requerimientos docentes de Chile, y requiere de buenos procesos para su acreditación. Pero, en cuanto a recursos, mejorar la formación docente principalmente sobre la base de convenios de desempeño estrechamente circunscritos en su forma, es premiar la capacidad técnica de formular proyectos y no reconocer la complejidad de elementos que entran a jugar en la formación y que no se expresan en puntajes ni en medidas discutibles de valor agregado. Caminar hacia una institucionalidad que apoye, regule, monitoree, investigue e informe a los programas de formación docente, como lo recomendó el Consejo Asesor Presidencial (2006), constituiría una acción positiva que instalaría más allá de los distintos gobiernos, un referente importante para la formación docente.

\section{REFERENCIAS BIBLIOGRÁFICAS}

Ávalos, B. (2002). Profesores para Chile. Historia de un Proyecto. Santiago de Chile: Ministerio de Educación.

Ávalos, B. y Matus, C. (2010). La formación inicial docente en Chile desde una óptica internacional: Informe nacional del estudio internacional IEA TEDS-M. Santiago de Chile: Ministerio de Educación.

Barber, M., \& Mourshed, M. (2007). How the world's best performing systems come out on top. Londres: McKinsey \& Co.

Berliner, D. (2014). Exogenous variables and value-added assessments: A fatal flaw. Teachers College Record, vol.116, n.1. Última visita 31 de mayo de 2014. Recuperado desde http://www. tcrecord.org/content.asp?contentid=17293

Centro de Estudios de Políticas y Prácticas en Educación. (2012). Proyecto de Ley que establece un puntaje de ingreso mínimo para carreras de pedagogía: algunas consideraciones. Notas para educación, n.12. Recuperado desde http://www.ceppe.cl/images/stories/recursos/notas/Notas_ Educacin_Septiembre_OK.pdf 
Consejo Asesor Presidencial para la Calidad de la Educación. (2006). Informe Final. Santiago. Última visita 27 mayo de 2014. Recuperado desde http://mt.educarchile.cl/MT/jjbrunner/archives/ libros/ConsejoAsesor/Inf_def.pdf

Conway, P.F. (2013). Cultural flashpoints: The politics of teacher education reform in Ireland. Educational Forum, vol.77, n.1, 51-72. doi: 10.1080/00131725.2013.739021

Cox, C., Meckes, L. y Bascopé, M. (2010). La institucionalidad formadora de profesores en Chile en la década del 2000: Velocidad del Mercado y Parsimonia de las Políticas. Pensamiento Educativo, vols.46-47, 205-245.

Domínguez, M., Bascopé, M. Meckes, L. y San Martín, E. (2012). ¿Producen mejores resultados las carreras de Pedagogía Básica con más años de acreditación? Estudios Públicos, n.128, 1-59.

Elige Educar. (2012). Propuestas Para Una Carrera Docente. Santiago: Elige Educar. Centro de Políticas Públicas UC.

Ell, F., \& Grudnoff, L. (2013). The politics of responsibility: Teacher education and 'persistent underachievement'. Educational Forum, vol.77, n.1, 73-86. doi: 10.1080/00131725.2013.739023

Furlong, J. (2006). New Labour and teacher education: the end of an era. Oxford Review of Education, vol.31, n.1, 119-134. doi: 10.1080/0305498042000337228

Furlong, J. (2013). Globalisation, neo-liberalism and the reform of teacher education in England. Educational Forum, vol.77, n.1, 28-50. doi 10.1080/00131725.2013.739017

García Huidobro, J. (2011). La política docente hoy y la formación de profesores. Docencia, n.43, $12-22$.

Ginsberg, R., \& Kingston, N. (2014). Caught in a vise: The challenges facing teacher education in an era of accountability. Teachers College Record, vol.116, n.1. Última visita 22 de abril de 2004. Recuperado desde http://www.tcrecord.org

Glazerman, S., Loeb, S., Goldhaber, D., Seiger, D., Raudenbush, S., \& Whitehurst, G. (2010). Evaluating Teachers: The Important Role of Value-Added. Brown Center on Educational Policy. Washington, DC: The Brookings Institute.

Haertel,E. (2013). How is testing supposed to improve schooling? Measurement: Interdisciplinary Research Perspectives, vol.11, n.1-2, 1-18. doi: 10.1080/15366367.2013.784165

Konstantopoulos, S. (2014). Teacher effects, value-added models, and accountability. Teachers College Record, vol.116, n.1. Última visita 31 de mayo de 2014. Recuperado desde http://www. tcrecord.org/content.asp?contentid $=17290$

Ministerio de Educación. (2009). Establece Ley General de Educación. Ley No 20.370. Biblioteca del Congreso Nacional. Ley Chile. Recuperado desde http://www.leychile.cl/ Navegar/?idNorma $=1006043 \&$ idParte $=0$

Meckes, L., \& Bascopé, M. (2012). Uneven distribution of novice teachers in the Chilean primary school system. Education Policy Analysis Archives, vol.20, n.20. Última visita 23 de mayo de 2014. Recuperado desde http://epaa.asu.edu/ojs/article/view/1017

Meyer, H-D. (2013). OECD's PISA: A tale of flaws and hubris. Teachers College Record. Última visita 20 de Diciembre de 2013. Recuperado desde www.tcrecord.org/

Ministerio de Educación. (2014). ¿Qué es un Convenio de Desempeño? Última visita 31 de mayo de 2014. Recuperado desde http://www.mece2.com/index2.php?id_seccion=3605\&id_ portal=59\&id_contenido=14986

Ministerio de Educación de Chile. (2005). Informe Comisión Formación Inicial Docente. Santiago de Chile: Serie Bicentenario.

National Council on Teacher Quality [NCTQ]. (2013). Teacher Prep Review: A Review of the Nation's Teacher Preparation Programs. National Council on Teacher Quality. Última visita 26 de mayo de 2014. Recuperado de www.nctq.org/teacherPrep

New Zealand Teachers Council. (2010). Approval, review, and monitoring processes and requirements for initial teacher education programmes. Wellington: author.

OCDE. (2005). Teachers Matter: Attracting, Developing and Retaining Effective Teachers. 


\section{Ediciones OCDE.}

O'Neill, J. (2012). Rationality and rationalisation in teacher education policy discourse in New Zealand. Educational Research, vol.54, n.2, 225-237.

UNESCO, OIT. (2008). Recomendación Conjunta de la OIT y UNESCO Relativa a la Situación del Personal Docente (1966) y Recomendación de la UNESCO Relativa a la Condición Docente de Enseñanza Superior (1997). París: UNESCO.

Panel de Expertos para una Educación de Calidad. (2010). Informe Final. Propuestas para Fortalecer La Profesión Docente en el Sistema Escolar Chileno. Santiago: Ministerio de Educación. Última visita 9 de Julio de 2010. Recuperado desde http://www.mineduc.cl/biblio/ documento/201007091211380.Informe\%20final.pdf

Paredes, R., Bogolasky, F., Cabezas, V., Rivero, R. y Zahri, M. (2013). Los determinantes del primer trabajo para profesores de Educación Básica en la Región Metropolitana. FONIDE F611105. Última visita 10 de Agosto de 2013. Recuperado desde http://centroestudios.mineduc.cl/index. $\mathrm{php} ? \mathrm{t}=96 \& \mathrm{i}=2 \& \mathrm{cc}=2180 \& \mathrm{tm}=2$

Pedraja, L., Araneda, C., Rodríguez, E. y Rodríguez, J. (2012). Calidad en la formación inicial docente: Evidencia empírica de las universidades chilenas. Formación Universitaria, vol.5, n.4, 15-26.

Rizvi, F., \& Lingvard, B. (2010). Globalizing Education Policy. Abingdon, Oxford: Routledge.

Toledo, G. (2009). Los profesores: ¿hacen la diferencia? Una aproximación desde la beca de alumnos destacados en pedagogía. Seminario de Título Ingeniero Comercial, Mención Economía, Universidad de Chile.

Valenzuela, J.P. y Sevilla, A. (2012). La movilidad de los nuevos profesores chilenos: los años que vivimos en peligro. Documento de trabajo.Centro de Investigación Avanzada en Educación. Universidad de Chile.

Zeichner, K. (2010). Competition, economic rationalization, increased surveillance, and attacks on diversity: Neo-liberalism and the transformation of teacher education in the US. Teaching and Teacher Education, vol.26, n.8, 1544-1552. 